\title{
Pre-synaptic control of remote fear extinction in the neocortex
}

\author{
Gisella Vetere ${ }^{1,2}$, Leonardo Restivo ${ }^{1,2+}$ and Martine Ammassari-Teule ${ }^{1,2 *}$ \\ CNR-National Research Council of Italy, Cell Biology and Neurobiology Institute, Rome, Italy \\ 2 Santa Lucia Foundation, Experimental Neurology Unit, Rome, Italy
}

\author{
Edited by: \\ Serge Laroche, Centre National de la \\ Recherche Scientifique and \\ University Paris-Sud, France \\ Reviewed by: \\ Jonathan L. C. Lee, Birmingham \\ University, UK \\ Guillaume Ferreira, Institut National \\ de la Recherche Agronomique, \\ France \\ *Correspondence: \\ Martine Ammassari-Teule, \\ CNR-National Research Council of \\ Italy, Cell Biology and Neurobiology \\ Institute, Santa Lucia Foundation, \\ Experimental Neurology Unit, \\ Via del Fosso di Fiorano 64, \\ 00143 Rome, Italy. \\ e-mail: martine.teule@cnr.it \\ ${ }^{\dagger}$ Present address: \\ Leonardo Restivo, Program in \\ Neurosciences and Mental Health, \\ Hospital for Sick Children, Toronto, \\ ON, Canada.
}

Consolidation of remote memory enhances immediate early genes induction (IEGs), augments the expression of the pre-synaptic growth associated protein-43 (GAP-43), and increases the density and size of dendritic spines in anterior cingulate (aCC) and infra-limbic (ILC) cortices. Remote memory extinction, however, does not uniformly alter consolidation-induced structural changes. In the aCC, the density, but not the size, of spines is reset to pseudo-conditioning levels while novel thin spines are formed in the ILC. Whether IEGs and GAP-43 also undergo region-specific changes upon remote memory extinction is undetermined. Here we confirm in the same batch of mice that c-Fos induction and GAP-43 expression are increased in both the aCC and the ILC 36 days after contextual fear conditioning. We then show that, in both regions, remote memory extinction is associated with decrease of c-Fos induction but no change in GAP-43 expression thus revealing similar, although protein-specific, pre-synaptic adaptations in aCC and ILC neurons. These observations, in addition to our previous report of region-specific post-synaptic structural changes, disclose a complex pattern of extinction-driven neocortical alterations suitable to support erasure or reinstatement of fear according to the environment demand.

Keywords: remote fear consolidation, remote fear extinction, anterior cingulate cortex, infra-limbic cortex, c-Fos, GAP-43

\section{INTRODUCTION}

Consolidation of remotely acquired memories require protein synthesis and structural re-arrangements in the neocortex, Mapping expression of activity-regulated immediate early genes (IEGs) or plasticity/cytoscheletal proteins several weeks after training revealed a parallel increase in c-Fos, and Zif induction (Frankland et al., 2004; Frankland and Bontempi, 2005) and in the pre-synaptic growth associated protein-43 (GAP-43) expression (Maviel et al., 2004) in anterior cingulate (aCC) and/or infra-limbic (ILC) cortices. In the same regions, post-synaptic alterations including dendritic spine formation (Restivo et al., 2009; Vetere et al., 2011a) and NMDA receptors enhancement (Takehara-Nishiuchi et al., 2006) were also detected in the conditioned mice.

Adaptation to varying environmental inputs implicates that even representations durably stored in memory can be modified throughout the individual's life and, depending on whether inputs are coherent or in conflict with the original representation, memories will be updated (Winocur et al., 2007; Lee, 2009) or extinguished (Quirk and Mueller, 2008). Although the pathological implications of abnormally long lasting memories, especially fear memories, can be as severe as those resulting from defective memory consolidation, the neural adaptations underlying remote extinction are poorly understood.
We recently reported that, although aCC and ILC neurons show a similar increase in spine density and size upon remote consolidation, these neurons undergo opposed remodeling upon remote extinction (Vetere et al., 2011b). In the aCC, the density, but not the size, of spines is reset to pseudo-conditioning levels while novel thin spines are formed in the ILC. Here we present data demonstrating that, differently from the dendritic spines which undergo region-specific changes, remote extinction decreases c-Fos induction and leaves unchanged GAP-43 expression in aCC and ILC neurons upon the remote memory test. Our findings therefore reveal that remote memory extinction promotes a complex pattern of neocortical adaptations which might support erasure or reinstatement of fear according to the environment demand.

\section{MATERIALS AND METHODS CONTEXTUAL FEAR CONDITIONING AND EXTINCTION}

C57/BL6 male mice $(N=33)$ were handled in the experimental room for 3 days to reduce apparatus-independent emotional reactions. On the fourth day, they were introduced in the conditioning chamber made of Plexiglas transparent walls $(28 \times 28 \times 10 \mathrm{~cm})$ inserted in an insulated box (TSE Systems $\mathrm{GmBH}$, Germany). The training schedule consisted in a $2 \mathrm{~min}$ habituation period followed by five foot-shocks $(0.7 \mathrm{~mA}, 2 \mathrm{~s})$ 
delivered at $1 \mathrm{~min}$ intervals. Mice were brought back to their home cage $1 \mathrm{~min}$ after the last foot-shock. Mice undergoing pseudo-training $(N=10)$ were left in the conditioning chamber for an equivalent time $(7 \mathrm{~min}$ ) without any foot-shock was delivered. Remote fear memory was assessed 36 days later by exposing half of the trained mice $(N=12)$ and the pseudotrained mice for $5 \mathrm{~min}$ to the experimental context. The other trained mice $(N=11)$ were returned to the chamber from day 32 to day 35 for a $7 \mathrm{~min}$ session/day to generate extinction, and then tested for remote memory on day 36 . Behavior during conditioning, extinction, and remote testing was recorded by means of a video camera mounted $60 \mathrm{~cm}$ above the ceiling of the cage and connected to a computer equipped with the Ethovision software (Noldus. Netherlands). The time spent freezing (absence of all but respiratory movements) was used to score fear memory and Z-projection of motor activity images were processed by imagej and Osirix software to visualize group-specific motor activity patterns during remote testing. The experiments were carried out in accordance with the guidelines provided by the European Communities Council Directive of 24 November 1986 (86/609/EEC).

\section{c-Fos AND GAP-43 IMMUNOCYTOCHEMISTRY AND IMAGING}

Mice were transcardially perfused $90 \mathrm{~min}$ after the completion of remote testing. Brains were removed, left in paraformaldheide (4\%) for $24 \mathrm{~h}$, and placed in phosphate-buffered 30\% sucrose for cryo-protection. Brains were sectioned using a cryostat at a thickness of $30 \mu \mathrm{m}$, the sections were collected and then used for c-Fos immunocytochemistry or GAP-43 immunofluorescence detection. Cortical regions were anatomically delimited using the atlas of Franklin and Paxinos (2001) Quantification of c-fos positive cells and GAP-43 expression levels was performed at bregma ranging from 1.10 to $0.02 \mathrm{~mm}$ for the aCC, and at bregma ranging from 1.94 to $1.54 \mathrm{~mm}$ for the ILC (Figure 2A).

\section{c-Fos immunocytochemistry}

c-Fos immunocytochemistry was performed using c-Fos-specific (1:20000; Calbiochem) primary rabbit polyclonal antibodies. A biotinylated goat anti-rabbit (1:2000; Chemicon) was used as secondary antibody. Staining was revealed using the avidin-biotin peroxidase method (ABC kit; Vectastain) coupled to diaminobenzidine as chromogen. Quantitative analyses of c-Fos positive nuclei were performed by means of a computerized imageprocessing system coupled to an optical microscope, and c-Fos labeled cells in layers II/III of aCC and ILC were counted using the optical fractionator method. Each counting frame $(50 \times 50 \mu \mathrm{m})$ was placed at an intersection of the lines forming a virtual grid which was moved within the region of interest. The $x-y$ stages of the microscope were automatically managed by the Neurolucida software (Microbrightfields, USA) that allowed to scan the regions of interest by shifting from one counting frame to the successive one. c-Fos labeled cells were counted using an oil-immersion objective (63X/1.40N.A.). Cells were included in the measurements only when they came into focus and their outline was well-defined. An average of 80 frames in the aCC and 50 frames in the ILC were counted. Single animal values were averaged to give the final means of the CONS (aCC: $N=7$; ILC:
$N=6$ ), the CONS + EXT (aCC: $N=7$; ILC: $N=6$ ) and the PSEUDO ( $N=6$ for both regions) groups.

\section{Immunofluorescence detection of GAP-43 expression}

Immunofluorescence detection of GAP-43 expression within the II/III layers of the aCC and ILC cortices was performed using primary rabbit polyclonal antibody GAP-43 (1:1000; Abcam), NeuN antibodies (1:500; Chemicon), and a fluorescein isothiocyanate (FITC)-conjugated goat anti-rabbit secondary antibody (1:500, Jackson ImmunoResearch) coupled with 4,6diamidino-2-phenylindole (DAPI) staining to visualize neurons nuclei. Densitometric analyses of fluorescence microscopy images were performed. After background subtraction, cell-associated to GAP-43 signals were quantified by manually outlining of individual neurons labeled by NeuN staining and measuring cell-associated fluorescence intensity with the Image software (http://rsb.info.nih.gov/ij/). The ratio, F/A, defines mean fluorescence of individual cells (F) normalized to total cellular surface (A). Quantification was performed in six animals per group (total of 130 cells per animal). Immunostained neurons were counted bilaterally using a minimum of four sections per animal. As for c-Fos immunocytochemistry, single animal values were averaged to give the final means of the CONS ( $N=6$ for both regions), the CONS $+\operatorname{EXT}(N=6$ for both regions $)$ and the PSEUDO $(N=6$ for both regions) groups.

All qualitative analyses were conducted blind to the animal's experimental group assignment.

\section{STATISTICAL ANALYSES}

Differences in freezing behavior between CONS, CONS + EXT, and PSEUDO mice during conditioning and remote testing were estimated by means of a One-Way ANOVA with the factor group as the between-factor. In the CONS + EXT group, the freezing response across extinction days was estimated by means of a OneWay ANOVA with the factor day as the within factor. Differences in c-Fos and GAP-43 expression upon remote testing were estimated by means of a Two-Way ANOVA with group (PSEUDO, CONS, CONS + EXT) and region of interest (ROI: aCC, ILC) as main factors. Post-hoc paired comparisons were carried out where necessary using the Bonferroni test.

\section{RESULTS}

\section{BEHAVIOR}

Behavioral experiments were run using the same contextual fear conditioning, remote extinction and remote memory test paradigms we set up for previous experiments aimed at analyzing changes in the density and size of dendritic spines were analyzed (Vetere et al., 2011b). Histograms depicting the freezing scores of CONS, CONS + EXT, and PSEUDO mice in each experimental condition are shown in Figure 1A while representative motor activity patterns during remote memory testing are shown in Figure 1B. The ANOVA performed on the conditioning data first revealed a main effect of group $\left[F_{(2,30)}=28.24\right.$, $P<0.001]$ with post-hoc pair comparisons indicating that the shocked mice (CONS and CONS + EXT) exhibited stronger freezing compared to the non-shocked mice (PSEUDO vs. any shocked group, $P<0.001)$. Conditioned mice re-exposed to the 
A

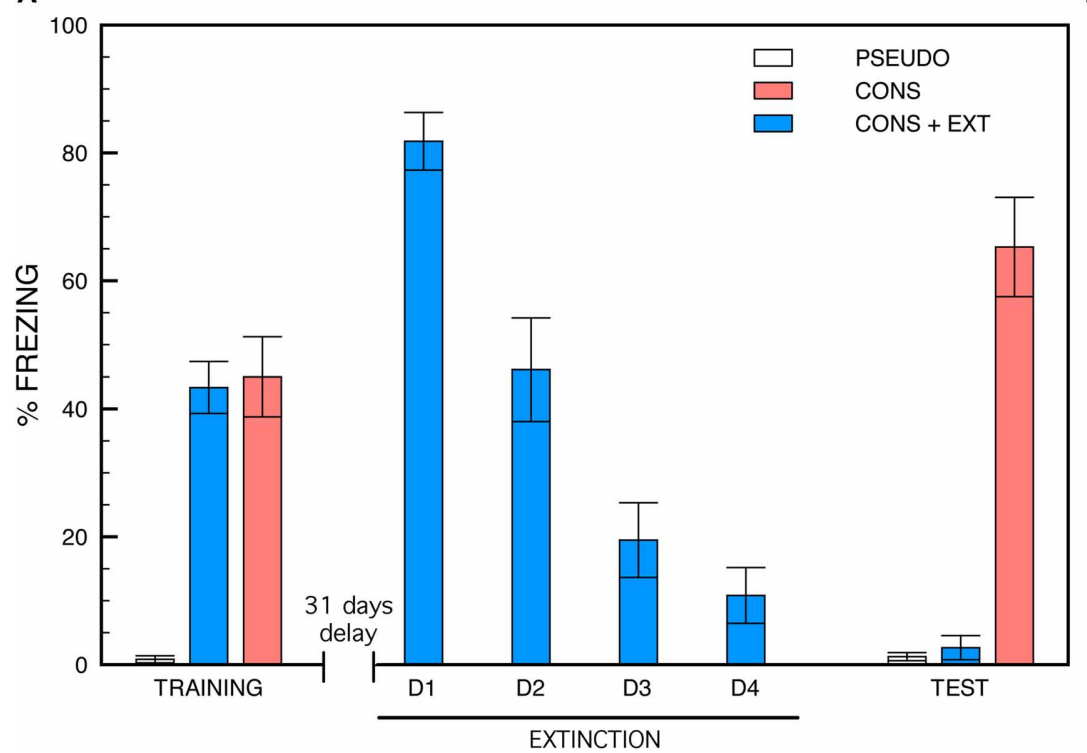

B
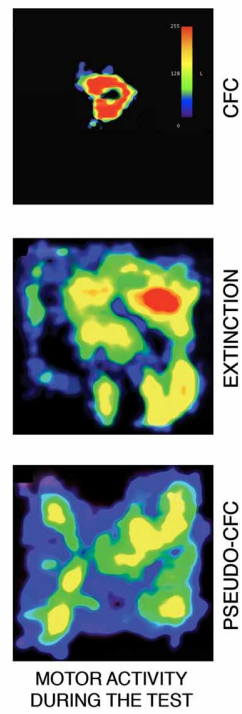

FIGURE 1 | Freezing behavior and motor activity patterns. (A) Freezing behavior during contextual fear conditioning, remote extinction and remote memory testing. During the conditioning, mice exposed to context-shock pairings (CONS and CONS + EXT) show robust freezing compared to non-shocked (PSEUDO) mice [group effect, $\left(F_{(2,30)}=28.24, P<0.001\right)$; CONS or CONS + EXT vs. PSEUDO, $P<0.001$ for each pair comparison). Remote fear extinction was generated by re-exposing half of the conditioned mice to the safe conditioning chamber from day 32 to day 35 . These mice (CONS + EXT) exhibited a progressive decrease in their freezing response across re-exposure days [effect of days, $\left(F_{(3,40)}=29.37, P<0.001\right)$ ].
Remote memory testing was run on day 36 . The conditioned mice that were not subjected to remote extinction (CONS) showed intense freezing compared to conditioned extinguished (CONS + EXT) and pseudo-conditioned (PSEUDO) mice $\left[F_{(2,30)}=54.71, P<0.001\right]$ which showed virtually no freezing (CONS + EXT vs. PSEUDO: $P>0.5$ ). (B) Representative motor activity patterns recorded during remote testing. Density plots for grouped data showing the locations where mice remained motionless within the conditioning chamber (color scale represents how long mice stayed at each location). CONS + EXT and PSEUDO mice show intense exploration of the conditioning chamber compared to CONS mice. safe chamber (CONS + EXT) exhibited a progressive decrease in their freezing response across re-exposure trials (effect of days, $\left[F_{(3,30)}=29.37, P<0.001\right]$. Then, the ANOVA performed on remote testing data still revealed a main effect of the group factor $\left[F_{(2,30)}=54.71, P<0.001\right]$. Post-hoc pair comparisons, however, revealed that the freezing scores of the CONS mice were significantly higher than those of the CONS + EXT and the PSEUDO mice $(P<0.001$ for each comparison) while no difference was found between the freezing scores of the CONS + EXT and the PSEUDO mice $(P>0.5)$ which showed virtually no freezing.

\section{C-Fos POSITIVE CELLS AND GAP-43 EXPRESSION LEVELS}

Statistical analyses of c-Fos positive cells and GAP-43 expression levels were performed on the raw data collected in the aCC and the ILC of CONS, CONS + EXT, and PSEUDO mice $90 \mathrm{~min}$ following remote testing. Histograms depicting the percentage increase of c-fos positive cells in the CONS and CONS + EXT groups relative to the PSEUDO group, and group representative c-Fos immunostained aCC and ILC sections are shown in Figure 2B. The ANOVA performed on these data revealed a significant group effect $\left[F_{(2,32)}=10.88, P<0.001\right]$ but no significant effect of ROI $\left[F_{(1,32)}=2.04, P=0.16\right]$ or of the ROI $\times$ group interaction $\left[F_{(2,32)}=0.01, P=0.98\right]$. Post-hoc comparisons then showed that, in both regions, $c$-fos positive cells were significantly more numerous in the CONS group than in the PSEUDO and the CONS + EXT group $(P<0.001$ for each comparison). No significant difference was found in any region between $\mathrm{c}$-fos positive cells counted in the PSEUDO and the CONS + EXT group thus indicating that extinction reset c-fos levels to those of non-extinguished pseudo-trained mice. Histograms depicting GAP-43 expression levels in the CONS and CONS + EXT groups relative to the PSEUDO group, and group representative GAP-43 immunostained/fluorescent aCC and ILC sections are shown in Figure 2C. The ANOVA performed on these data revealed a significant group effect $\left[F_{(2,30)}=15.67\right.$, $P=0.001]$ but no significant of ROI $\left[F_{(1,30)}=1.72, P=0.19\right]$ or of the ROI $\times$ group interaction $\left[F_{(2,30)}=0.09, P=0.90\right]$. Post-hoc comparisons then showed that, in both regions, GAP43 levels were significantly lower in the PSEUDO mice than in the CONS and CONS + EXT mice $(P<0.001$ for all comparisons). No significant difference was found in GAP-43 levels detected between CONS and CONS + EXT mice.

\section{DISCUSSION}

Contextual fear memory and extinction have been predominantly investigated at training-to-test intervals compatible with recent memory formation and, at those intervals (24-72 h), a circuit linking the amygdala, the hippocampus and the medial prefrontal cortex was identified. Within this circuit, the hippocampus and the amygdala were found to be involved in the formation of contextual fear memory while neocortical regions including the aCC 
A
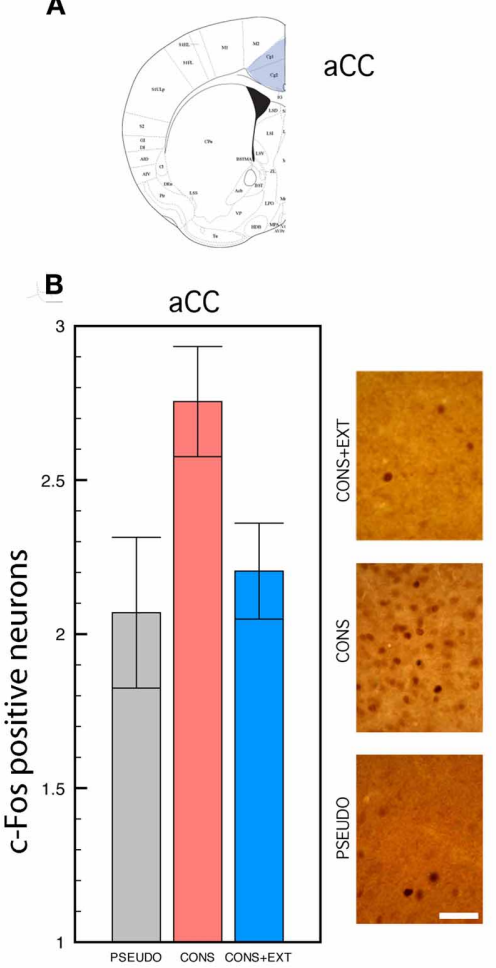

C
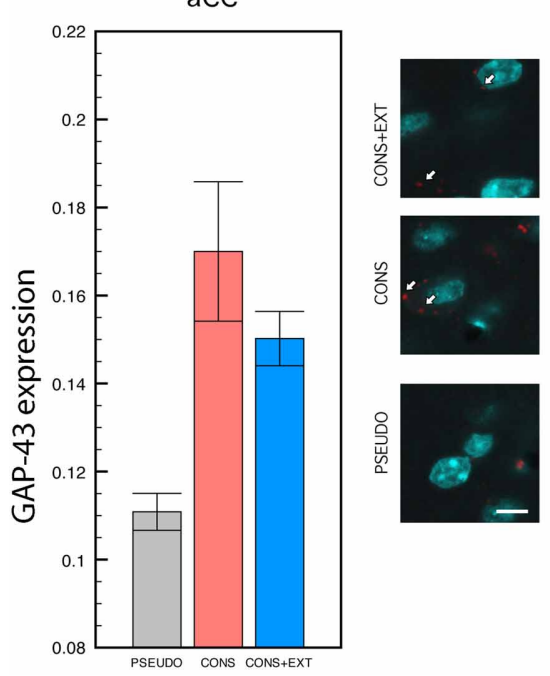

FIGURE 2 | c-Fos immunocytochemistry and GAP-43

immunofluorescence. Statistical comparisons were performed on the raw data. (A) Anatomical delimitation of anterior cingulate $(\mathrm{aCC})$ and infra-limbic (ILC) cortices based on Franklin and Paxinos (2001). (B) c-Fos positive cells counted in the aCC and the ILC of PSEUDO, CONS, and CONS + EXT mice after remote memory testing. In both regions, c-Fos positive cells were significantly more numerous in the CONS group than in the PSEUDO and the CONS + EXT groups among which no difference was found [significant group effect: $\left(F_{(2,32)}=10,88, P<0.001\right)$, CONS vs. PSEUDO or CONS + EXT, $P<0.001$ for each pair comparison; non-significant ROI effect:

\section{ILC}

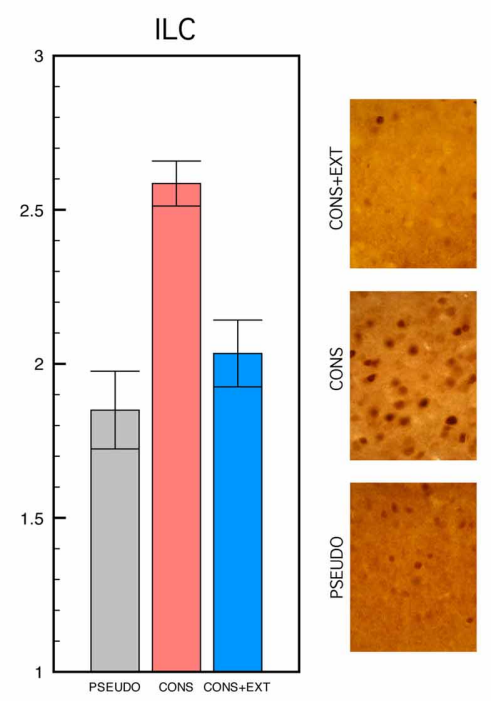

ILC

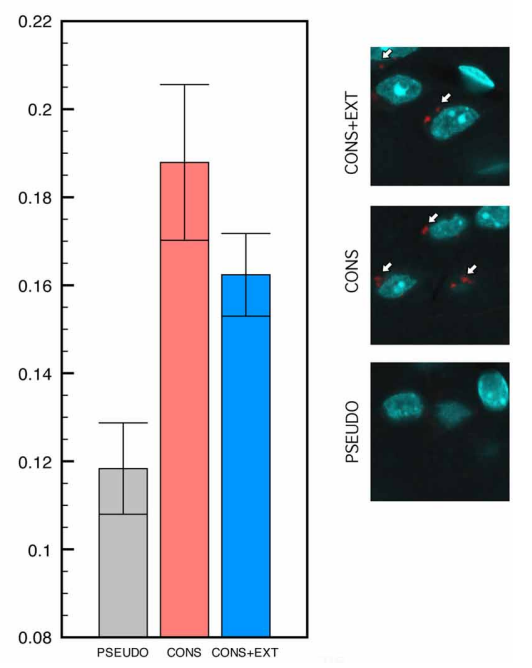

$\left(F_{(1,32)}=2.04, P=0.16\right)$; non-significant $\mathrm{ROI} \times$ group interaction:

$\left.\left(F_{(2,32)}=0.01, P=0.98\right)\right]$. Scale bar: $50 \mu \mathrm{m}$. (C) Densitometric analyses of GAP-43 signal in individual neurons labeled with NeuN after remote memory testing. The GAP-43 signal values were significantly lower in the PSEUDO group than in the CONS and the CONS + EXT group among which no difference was found [group effect: $\left(F_{(2,30)}=15,67, P=0.001\right)$, PSEUDO vs. CONS or CONS + EXT, $P<0.001$ for each pair comparison; ROI effect: $\left(F_{(1,30)}=1,72, P=0.19\right) ; \mathrm{ROI} \times$ group interaction: $\left(F_{(2,30)}=0.09\right.$, $P=0.90)$ ]. Scale bar: $10 \mu \mathrm{m}$. White arrows indicate the GAP-43 immunofluorescent labeling. 
and the ILC were implicated in the extinction of contextual fear (see Orsini and Maren, 2012 for a review). It is, however, well established that the hippocampus is a time-limited node in the storage of contextual fear memory. Data showing that the formation of recent contextual fear memory enhances c-Fos, zif, GAP-43, and spine density in the hippocampus while, at remote time-points, a similar pattern of protein expression and structural remodeling is observed in the aCC and the ILC, indicate that remote memory traces are established in the neocortex. It turns out that, at remote time-points, medial prefrontal cortex regions might contemporaneously govern memory extinction and memory storage, underlining the crucial need for elucidating which elements in the neocortical circuitry might be specific to each function.

Considering that the aforementioned activity-dependent genes and GAP-43 analyses were not all performed at exactly the same remote consolidation time-points and/or cortical regions, the first part of our study consisted in recapitulating these observations in the same batch of mice to successively provide parallel description of their modification upon remote extinction. Our consolidation data collected at a training-to-test interval of 36 days revealed a significant increase in c-Fos induction and GAP-43 expression in both the aCC and the ILC of trained mice vs. pseudo-trained mice that were only exposed to context, the actually novel findings being that GAP-43, until now examined in the aCC following remote consolidation of spatial discrimination learning (Maviel et al., 2004) is enhanced in the aCC and the ILC upon remote consolidation of contextual fear. Thus, our findings definitely establish that (1) pre-synaptic proteins regulating neuronal activity or cytoskeletal organization and (2) post-synaptic structural changes including the number and the dimension of spines in the apical dendrite compartment (Vetere et al., 2011b), go through a parallel increase in the aCC and the ILC during remote fear memory formation.

Mice undergoing remote memory extinction were returned to the conditioning apparatus for an $8 \mathrm{~min}$ session during posttraining days $32-35$, and then for a 5 min remote memory test on post-training day 36. This protocol generated a progressive decrease in their freezing response until levels which, during remote testing, were not different from those of non-extinguished pseudo-trained mice. Mapping c-Fos activity in the aCC and the ILC following the remote memory test then revealed that suppression of freezing behavior in the CONS + EXT mice was associated with a decrease in c-Fos expression to the same level observed in non-extinguished pseudo-trained mice. Although comparing the c-Fos data of CONS + EXT mice to a group of PSEUDO mice repeatedly turned to the experimental context from days 32 to 35 might have been of a potential interest, our findings overall indicate that the c-fos induction status of ILC neurons do not differ between CONS + EXT and PSEUDO mice, two mice groups showing the same absence of freezing during remote memory testing. Our observations are therefore in contrast with recent extinction data showing that induction of c-Fos expression in the ILC remains elevated (Herry and Mons, 2004) or even more increased (Knapska and Maren, 2009) in mice exposed to extinguished CS in the extinction context. It is unlikely that differences between conditioning procedures, i.e., the absence of explicit CS in our protocol, account for this discrepancy. There is, in fact, no evidence that the formation of an auditory sensorial representation involves the ILC more than the formation of a contextual representation. In addition, the recent report that inactivating the ILC impairs place preference extinction (Groblewski et al., 2012) indicates that ILC involvement in memory extinction is task-independent. Differently, the fact that the extinction sessions were more numerous in our protocol than in those of Herry and Mons (2004) or Knapska and Maren (2009) might have contributed. Specifically, it could be that experiencing four, instead of one, extinction sessions before the remote memory test might have consolidated extinction learning and disengaged, as far as c-fos induction in ILC neurons is concerned, a cortical region previously involved in consolidation of remote memory.

The pre-synaptic protein GAP-43 is protein kinase C phosphorylation substrate controlling axonal growth cones and axon regeneration (Benovitz and Routtenberg, 1997). In adult animals, enhanced GAP-43 levels and/or phosphorylation are found in the hippocampus upon CFC training (Young et al., 2000, 2002) or spatial discrimination learning (Maviel et al., 2004) which also promotes the formation of dendritic spines (Restivo et al., 2009; Middei et al., 2010) therefore suggesting a role for this protein in activity-dependent neuronal remodeling. Thus, the observations that GAP-43 and spine density are concomitantly increased $36 \mathrm{~d}$ after the conditioning is consistent with the prediction that neurons expressing high GAP-43 levels are especially amenable to activity-dependent synaptic re-arrangements (Benovitz and Routtenberg, 1997). Surprisingly, upon extinction, the still high GAP-43 levels found in the aCC and the ILC are associated with aCC spine loss and ILC spine growth. This apparent contradiction can be resolved considering that LTD and LTP, that favor spine growth and spine elimination respectively (Nagerl et al., 2004; Zhou et al., 2004), induce a short-term increase and decrease of GAP-43 phophorylation in the CA1 field (Ramakers et al., 1999, 2000). Thus, high levels of GAP43 might be the marker of a capability of neurons to undergo activity-dependent structural changes, whatever the direction of the changes, while the phosphorylation state of the protein, which impacts on synaptic efficacy, would specify the direction of the change.

Altogether our results show that remote memory extinction drives similar, although protein-specific, pre-synaptic adaptations in aCC and ILC neurons. In both regions, induced c-Fos expression levels return to baseline while GAP-43 expression remains elevated. This means that the opposed structural changes we reported in these regions require that high GAP-43 expression levels persist. Importantly, the region-specific structural changes we detected upon remote extinction included the neo-formation of thin spines in the ILC and the persistence of large spines in the aCC (Vetere et al., 2011b). On the one hand, the presence of newly formed ILC synaptic contacts account for a reshaping of ILC connections coherent with a novel function this region might be involved in, and both earlier (Milad and Quirk, 2002) and recent (Ghazizadeh et al., 2012) data indicate that this function consist in the suppression of unreinforced actions. On the other, large spines are associated with lifelong memories (Yang et al., 2009) 
suggesting that their presence in the aCC might be crucial for reactivating extinguished fear memories. It is therefore apparent that the complex pattern of neocortical re-arrangements observed upon remote extinction warrants region-specific subtle adjustments of neuronal activity and connectivity enabling erasure or reinstatement of fear responses under appropriate environmental inputs.

\section{REFERENCES}

Benovitz, L. I., and Routtenberg, A. (1997). GAP-43, an intrinsic determinant of neuronal development and plasticity. Trends Neurosci. 20, 84-91.

Frankland, P. W., and Bontempi, B. (2005). The organization of recent and remote memories. Nat. Rev. Neurosci. 6, 119-130.

Frankland, P. W., Bontempi, B., Talton, L. E., Kaczmarek, L., and Silva, A. J. (2004). The involvement of the anterior cingulate cortex in remote contextual fear memory. Science 304, 881-883.

Franklin, K. B. J., and Paxinos, G. (2001). The Mouse Brain in Stereotaxic Coordinates, 2nd edn. San Diego, CA: Academic Press.

Ghazizadeh, A., Ambroggi, F., Odean, N., and Fileds, H. L. (2012). Prefrontal cortex mediates extinction of responding by two distinct neural mechanisms in accumbens shell. J. Neurosci. 32, 726-737.

Groblewski, P. A., Ryabinin, A. E., and Cunningham, C. L. (2012). Activation and role of the medial prefrontal cortex (mPFC) in extinction of ethanol-induced associative learning in mice. Neurobiol. Learn. Mem. 97, 37-46.

Herry, C., and Mons, N. (2004). Resistance to extinction is associated with impaired immediate early geme induction in medial prefrontal cortex and amygdala. Eur. J. Neurosci. 20, 781-790.

Knapska, E., and Maren, S. (2009). Reciprocal patterns of c-Fos expression in the medial prefrontal cortex and amygdala after extinction and renewal of conditioned fear. Learn. Mem. 16, 486-493.
Lee, J. L. (2009). Reconsolidation: maintaining memory relevance. Trends Neurosci. 32, 413-20.

Maviel, T., Durkin, T. P., Menzaghi, F., and Bontempi, B. (2004). Sites of neocortical reorganization critical for remote spatial memory. Science 305, 96-99.

Middei, S., Roberto, A., Berretta, N., Panico, M. B., Lista, S., Bernardi, G., Mercuri, N. B., Ammassari-Teule, M., and Nisticò, R. (2010). Learning descloses abnormal structural and functional plasticity at hippocampal synapses in the APP23 mouse model of Alzheimer's Disease. Learn. Mem. 17, 236-240.

Milad, M. R., and Quirk, G. J. (2002). Stimulation of infralimbic cortex simulates memory for extinction of conditioned fear. Soc. Neurosci. Abstr. 83.12.

Nagerl, U. V., Eberhorn, N., Cambridge, S. B., and Bonhoeffer, T. (2004). Bidirectional activitydependent morphological plasticity in hippocampal neurons. Neuron 44, 759-767.

Orsini, C. A., and Maren, S. (2012). Neural and cellular mechanisms of fear and extinction memory formation. Neurosci. Biobehav. Rev. doi: 10.1016/j.neubiorev.2011.12.014. [Epub ahead of print]

Quirk, G. J., and Mueller, D. (2008). Neural mechanisms of extinction learning and retrieval. Neuropsychopharmacology 33, 56-72.

Ramakers, G. M., Heinen, K., Gispen, W. H., and de Graan, P. N. (2000). Long term depression in the CA1 field is associated with transient decrease in pre- and postsynaptic PKC substrate phosphorylation. J. Biol. Chem. 275, 28682-28687.

Ramakers, G. M., McNamara, R. K., Lenox, R. H., and de Graan, P. N. (1999). Differential changes in the

\section{ACKNOWLEDGMENTS}

This work was supported by Regione Lazio "Sviluppo della Ricerca sul Cervello" (Martine Ammassari-Teulea) and Minitero della Salute, progetto finalizzato N 263/RF-2009-1536072 (Martine Ammassari-Teulea). The authors are grateful to Prof. Aryeh Routtenberg, Northwstern University Evanston, USA, for critical reading of the manuscript.

phosphorylation of the protein kinase $\mathrm{C}$ substrates myristoylated alanine-rich $\mathrm{C}$ kinase substrate and growth-associated protein-43/B-50 following Schaffer collateral longterm potentiation and long-term depression. J. Neurochem. 73, 2175-2183.

Restivo, L., Vetere, G., Bontempi, B., and Ammassari-Teule, M. (2009). The formation of recent and remote memory is associated with time-dependent formation of dendritic spines in the hippocampus and anterior cingulate cortex. J. Neurosci. 29, 8206-14.

Takehara-Nishiuchi, K., Nakao, K., Kawahara, S., Matsuki, N., and Kirino, Y. (2006). Systems consolidation requires postlearning activation of NMDA receptors in the medial prefrontal cortex in trace eyeblink conditioning. J. Neurosci. 26, 5049-5058.

Vetere, G., Restivo, L., Cole, C. J., Ross, P. J., Ammassari-Teule, M., Josselyn, S. A., and Frankland, P. W. (2011a). Spine growth in anterior cingulate cortex is necessary for the consoldaition of contextual fear memory. Proc. Natl. Acad. Sci. U.S.A. 108, 8456-8460.

Vetere, G., Restivo, L., Novembre, G., Aceti, M., Lumaca, M., and Ammassari-Teule, M. (2011b). Extinction partially reverts structural changes associated with remote fear memory Learn. Mem. $18,554-557$.

Winocur, G., Moscovitch, M., and Sekeres, M. (2007). Memory consolidation or transformation: context manipulation and hippocampal representations of memory. Nat. Neurosci. 10, 555-557.

Yang, G., Pan, F., and Gan, W. B. (2009). Stably maintained dendritic spines are associated with lifelong memories. Nature 462, 920-924.

Young, E., Cesena, T., Meiri, K. F., and Perrone-Bizzozero, N. I. (2002) Changes in Protein Kinase, C (PKC) activity, isozyme translocation, and GAP-43 phosphorylation in the rat hippocampal formation after single trial contextual fear conditioning paradigm. Hippocampus 12 , 457-464.

Young, E. A., Owen, E. H., Meiri, K. F., and Wehner, J. M. (2000). Alterations in hippocampal GAP-43 phosphorylation and protein levels following contextual conditioning. Brain Res. 860 , 95-103.

Zhou, Q., Homma, K. J., and Poo, M. M. (2004). Shrinkage of dendritic spines associated with longterm depression of hippocampal synapses. Neuron 44, 749-757.

Conflict of Interest Statement: The authors declare that the research was conducted in the absence of any commercial or financial relationships that could be construed as a potential conflict of interest.

Received: 29 March 2012; accepted: 08 June 2012; published online: 21 June 2012.

Citation: Restivo L, Vetere $G$ and Ammassari-Teule M (2012) Presynaptic control of remote fear extinction in the neocortex. Front. Behav. Neurosci. 6:34. doi: 10.3389/fnbeh.2012.00034 Copyright (c) 2012 Vetere, Restivo and Ammassari-Teule. This is an openaccess article distributed under the terms of the Creative Commons Attribution Non Commercial License, which permits non-commercial use, distribution, and reproduction in other forums, provided the original authors and source are credited. 